\title{
Kalman Filter Based Electromyographic Signal Suppression of Real-Time ECG Signal
}

\author{
Meng Chen ${ }^{1}$, Yizhou Zhong ${ }^{1}$, Huaiyu Zhu ${ }^{2}$, Yun Pan ${ }^{2}$, \\ ${ }^{1}$ Hangzhou Proton Technology Co., Ltd., Hangzhou, Zhejiang, China \\ ${ }^{2}$ College of Information Science \& Electronic Engineering, Zhejiang University, China
}

\begin{abstract}
Electromyographic (EMG) noise has a broad bandwidth overlapping on the ECG signal, which is hard to suppress. This research uses one-dimensional Kalman filter to remove EMG noise after preliminary filtering and QRS complex wave recognition of real-time ECG signal. In this research, the low pass and high-pass FIR filter are used firstly to suppress power line and high frequency interference. Then a median filter is used to delete baseline wander. A Kaiser window is also used to prevent spectrum leakage. After these pre-processing, the wavelet transform method is used to initially identify the R peaks, $Q$ peaks and S peaks. Since EMG noise is similar as white noise as to ECG, Kalman filter is suitable to remove $E M G$ in real time. We generate an EMG noise database by adding EMG noise from Noise Stress Test database to clean ECG data in MIT-BIH Arrhythmia Database. We test the ECG data in EMG noise database and 1475 ECG data collected by a portable ECG card. Without weakening the $R$ peaks, the EMG noise is suppressed successfully, while P-peaks can be automatically identified with the smooth signal, which helps to identify premature ventricular contraction $(P V C)$. The sensitivity and positive predict value $(+P)$ of $Q R S$ recognition and $P$ recognition of EMG noise database are all above $99 \%$.
\end{abstract}

\section{Introduction}

ECG signal is a weak bio-electricity signal, and can be influenced by baseline wander, power line interference, movement interference, electromyographic (EMG) signal and so on. The ongoing trend of long-term ECG monitoring techniques requires ambulatory and less obtrusive ECG signal collection devices. However, it comes at the cost of decreased signal quality. Some of the artefact problems can be suppressed by simple and frequency selected filters, such as baseline wander interference and power line interference.

The baseline wander is a low-frequency interference, while the power interference has a fixed frequency. There are various methods to eliminate these noises, such as finite impulse response (FIR) filtering, infinite impulse response (IIR) filtering, adaptive filtering, Fuzzy logic and wavelet transform. However, the EMG noise is difficult to be filtered, due to its considerable overlapping frequency spectrum with ECG [1,2]. EMG noise is a random signal and it varies from time to time. Many methods have been used to eliminate EMG from noised ECG signals. However, they usually result in limited suppression of EMG artefact but considerable reduction of sharp QRS wave amplitudes. Ivoylo et al proposed a dynamic low-pass filter for EMG noise suppression in ECG [3]. Sayyad et al used extended Kalman filter and extended Kalman smoother to eliminate EMG, but both methods distorted QRS observably [4]. Joy et al removed EMG noise by selecting the most suitable wavelet function based on the $5^{\text {th }}$ wavelet decomposition level [2]. Some researchers applied independent component analysis (ICA) and principle component analysis (PCA) to split EMG from ECG, but these methods do not perform well for single-channel ECG devices.

Kalman filter (KF) is an optimal state estimation process applied to a dynamic system that involves random perturbations [5-7]. The KF is a linear, unbiased, and minimum error variance recursive algorithm and can optimally estimate the unknown state of a system from noisy data taken at discrete real-time. While in prediction step, KF produces estimates of the current variables, along with their uncertainties. Once the outcome of next measurement is observed, these estimates are updated. KF has been widely used in many areas of industrial and government applications, such as video and laser tracking system, satellite navigation and biomedical signal processing $[5,6]$.

In this research, utilizing the time-frequency characterize of EMG signal, the authors proposed one dimensional $\mathrm{KF}$ to suppress EMG noise in singlechannel ECG signal. EMG interference can be regarded as transient Gaussian zero mean band-limited noise. KF is suitable to eliminate EMG noise after pre-processing filtering and initial QRS complex recognition.

\section{Methods}




\subsection{EMG noise database establishment}

While the MIT-BIH Noise Stress Test Database (NSTDB) only contain electrode motion artefact data, an EMG noise database is established to verify the effectiveness of KF in this research. The noised ECG recordings were created by the script nstdbgen- using several clean recordings, such as 100 and 101, from the MIT-BIH Arrhythmia Database (MITDB) [8], to which calibrated amounts of noise from record 'ma' were added using nst [9]. The detailed data information is in Table 1.

Table 1. The signal-to-noise ratios (SNRs) during the noisy segments of these records

\begin{tabular}{lllc}
\hline Record & SNR(dB) & Record & SNR(dB) \\
\hline $100 \mathrm{~m} 18$ & 18 & $100 \mathrm{~m} 24$ & 24 \\
$101 \mathrm{~m} 18$ & 18 & $101 \mathrm{~m} 24$ & 24 \\
$111 \mathrm{~m} 18$ & 18 & $111 \mathrm{~m} 24$ & 24 \\
$124 \mathrm{~m} 18$ & 18 & $124 \mathrm{~m} 24$ & 24 \\
$200 \mathrm{~m} 18$ & 18 & $200 \mathrm{~m} 24$ & 24 \\
$201 \mathrm{~m} 18$ & 18 & $201 \mathrm{~m} 24$ & 24 \\
$232 \mathrm{~m} 18$ & 18 & $232 \mathrm{~m} 24$ & 24 \\
$234 \mathrm{~m} 18$ & 18 & $234 \mathrm{~m} 24$ & 24 \\
\hline
\end{tabular}

\subsection{Pre-processing filtering}

To get an efficient signal process algorithm, the raw ECG signal is processed every 2 seconds and overlap 1 second to the next 2 seconds as to prevent QRS distortion. Then the windowed ECG signal will experience the following pre-processing. Firstly, the DC interference is eliminated by subtracting the mean value from each ECG samples. Secondly, a FIR low-pass filter is applied to remove the high-frequency and power line interference. Thirdly, a FIR high-pass filter is utilized to cancel the low-frequency noise. Then, the baseline wander is rectified by a median filter. At last, a 341-order Kaiser window is applied to prevent the spectrum leakage.

After the aforementioned filtering, wavelet threshold method is used to detect QRS complex at windowed ECG data [10]. What's more, the KF algorithm and $\mathrm{P}$ peak detection method are then applied. Figure 1 shows the entire process of the method proposed in this paper.

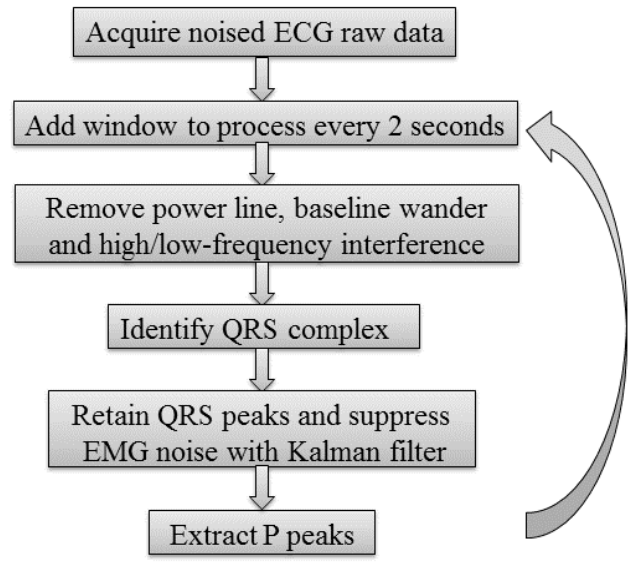

Figure 1. The flowchart of the real-time process of the ECG signal based on Kalman filter

\subsection{Kalman Filtering}

$\mathrm{KF}$ is an optimal filtering algorithm based on iterative calculation. The ECG signal can be assumed as a dynamic process and described by a dynamic autoregressive model. The dynamic equation and measurement equation can be constructed as:

$$
\begin{aligned}
& \mathrm{X}_{\mathrm{t}}=\phi_{\mathrm{t}, \mathrm{t}-1} \mathrm{X}_{\mathrm{t}-1}+\Gamma_{\mathrm{t}, \mathrm{t}-1} \mathrm{~W}_{\mathrm{t}-1} \\
& \mathrm{Z}_{\mathrm{t}}=\mathrm{H}_{\mathrm{t}} \mathrm{X}_{\mathrm{t}}+\mathrm{V}_{\mathrm{t}}
\end{aligned}
$$

Where $\mathrm{X}$ is the dynamic vector of ECG signal, $\mathrm{Z}$ is the measurement of pre-processed ECG signal, $t$ is the $t$-th iterative dynamic update, $\phi_{t, t-1}$ is the dynamic transition matrix and $\Gamma_{t, t-1}$ is the noise input transition matrix which are simplified as constants in later calculations, W is the process noise which can represent the EMG noise, and $\mathrm{V}$ is the measurement noise which can be assumed as white noise. The statistic characteristics of $\mathrm{W}$ and $\mathrm{V}$ can be assumed as $\mathrm{N}(0, \overline{\mathrm{Q}})$ and $\mathrm{N}(0, \overline{\mathrm{R}})$, respectively.

The iterative update of KF can be expressed as Figure 2 , where $X_{t}$ is the estimation of dynamic vector, $X_{t, t-1}$ is the estimate of one-step predictive value of dynamic vector, $\mathrm{P}_{t}$ is the error covariance matrix of dynamic vector which represents the errors in the estimate, $P_{t, t-1}$ is the one-step predictive value of error covariance matrix, and $\mathrm{K}_{\mathrm{t}}$ is the Kalman gain matrix. $\mathrm{X}_{1}$ and $\mathrm{P}_{1}$ are initial values of dynamic vector and error covariance matrix. $\mathrm{H}_{\mathrm{t}}$ is the conversion matrix to transform the covariance matrix of dynamic vector to the dynamic matrix and $R_{t}$ is the measurement covariance matrix which represents the errors of measurement. When calculating the one-step predictive value, the initial values become the previous 
values. Kalman gain equals to the ratio of the error of estimate to the sum of errors of estimate and measurement. In Figure 2, the filtering process can be divided into time update and measurement update stages in the update process which interconnect with each other.

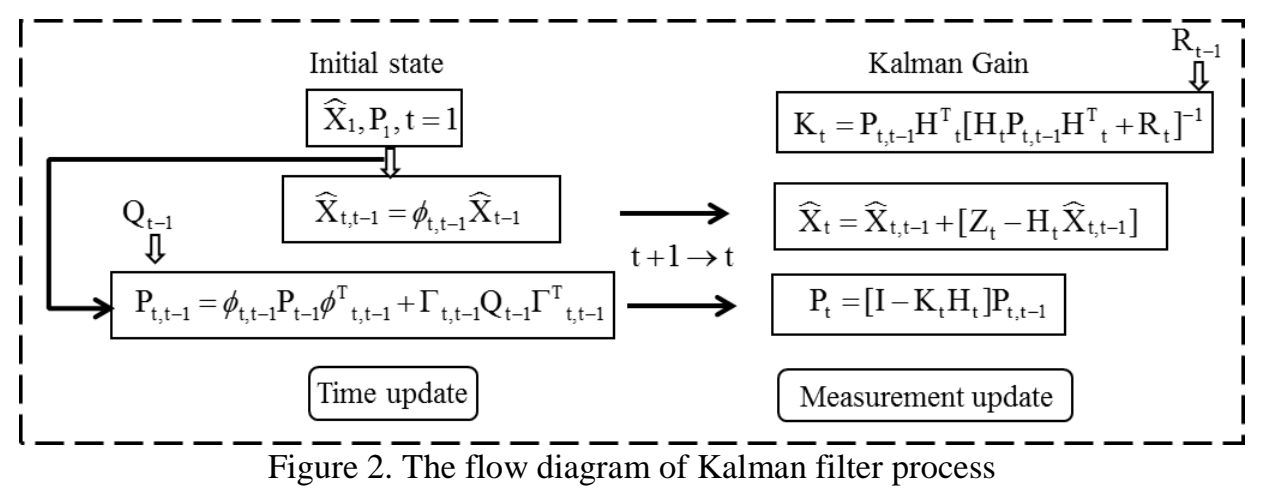

\section{Results}

The sensitivity (SE) and positive predict value $(+\mathrm{P})$ of QRS detection are $99.46 \%$ and $99.39 \%$ among 48 data in MITDB. And the SE and $+\mathrm{P}$ of QRS detection among EMG noise database is $99.71 \%$ and $99.64 \%$.

After the application of KF, the QRS complex remain unchanged and only the EMG noises between RR intervals are suppressed. Figure 3 clearly represents the effectiveness of KF, where the dotted line shows the noised data after pre-processing and the solid line is the final wave after KF processing. Figure 4 depicts a segment of raw data in MITDB, the same segment data overlapped with EMG noise as Figure 3, the signal after pre-processed filtering and the signal after KF processing. Figure 5 describes the EMG noise eliminated by the KF. The SNR of the noised ECG data is $18 \mathrm{~dB}$ and the data in Figure 5 is the same as that in Figure 3 and Figure 4.

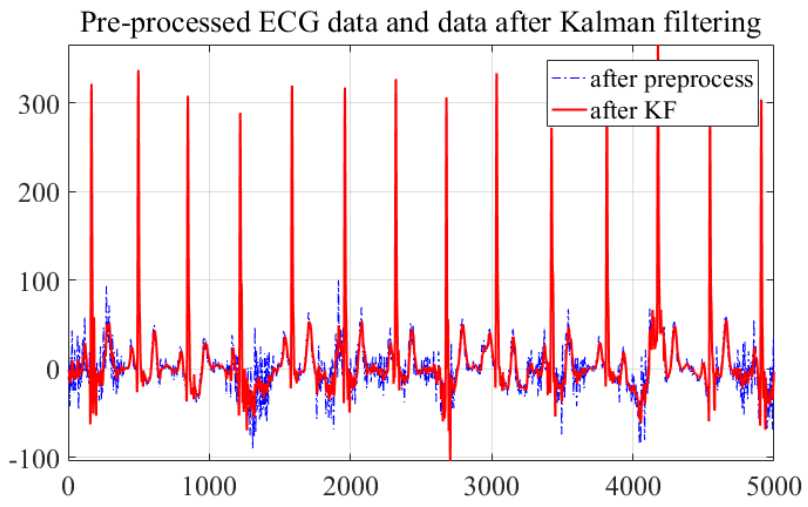

Figure 3. The dotted and solid lines designate the data after pre-processed filtering and data after $\mathrm{KF}$ respectively of noised \#101 data in MITDB

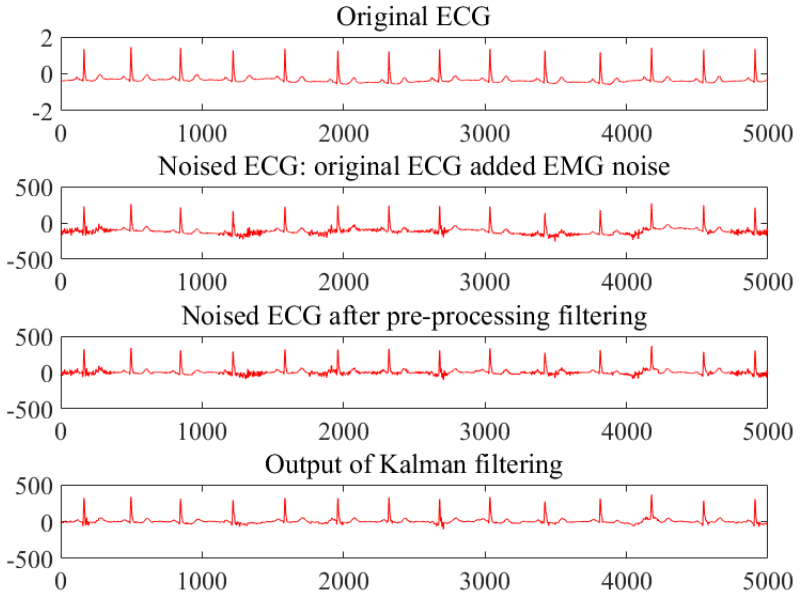

Figure 4. Simulation results of Kalman filter with EMG noise $(\mathrm{SNR}=18 \mathrm{~dB})$

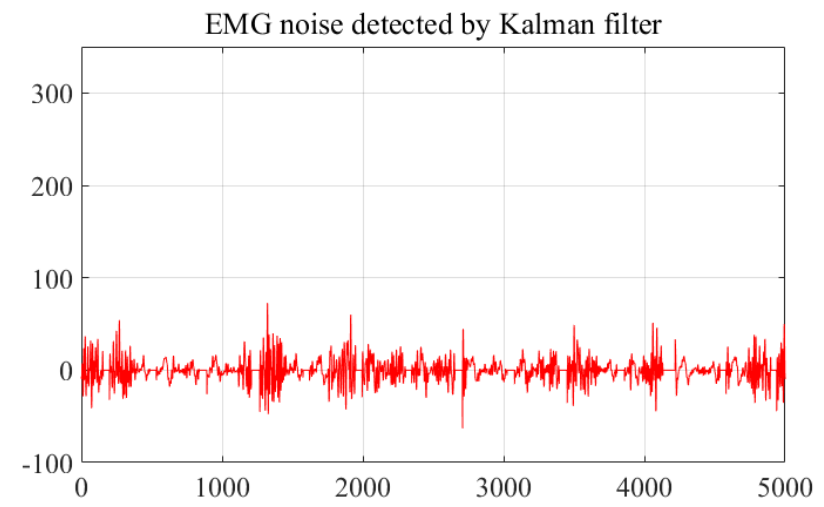

Figure 5. The EMG noise detected by Kalman filter

After the pre-processing and KF filtration, all the QRS peaks are reserved and remain in good shape. Then combing the threshold method with wing function, the $\mathrm{P}$ peaks are detected in the final processed ECG waves 
without EMG interference [3]. Figure 6 shows the R peaks and $\mathrm{P}$ peaks of the same segment identified by the algorithm, where $\mathrm{R}$ peaks are identified by wavelet threshold method. The R peaks and P peaks are marked in the pre-processed data, and plotted by circle and pentagram symbols respectively. The $\mathrm{SE}$ and $+\mathrm{P}$ of $\mathrm{P}$ peaks detection in EMG noise database are all above $99 \%$.

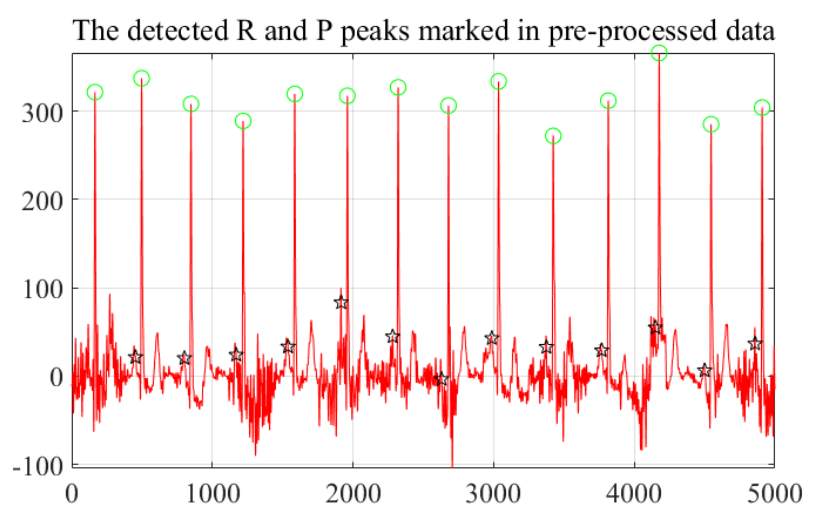

Figure 6 . The $\mathrm{R}$ peaks and $\mathrm{P}$ peaks are marked in the preprocessed data, and plotted by circle and pentagram symbols respectively

\section{Discussions and Conclusions}

In this paper, a KF based EMG noise suppression method is designed for the filtering of real-time ECG signals. EMG interference can be regarded as transient Gaussian zero mean band-limited noise. The onedimensional linear dynamic KF model is suitable for white noise elimination after pre-processing filtering and initial QRS complex recognition.

Before the application of KF, several other filters are used to remove high-frequency noise, low-frequency noise, powerline interference and baseline interference. Movement interference is not considered, since the data chosen from MITDB do not have obvious movement interference. The proposed algorithm not only suppresses EMG interference, but also keeps the QRS complex in good shape. The denoising result is showed in Figure 3-5. With the denoised ECG data, $P$ peaks are detected in this paper. The QRS and $\mathrm{P}$ peaks are extracted by using wavelet threshold and wing function threshold method respectively, both of which perform well.

In addition to the data in open database, we also acquired 1475 raw ECG data by applying a potable ECG card and evaluated the QRS identification algorithm using these data. However, these data were not annotated by experts and we will discuss the effectiveness of this algorithm on these data in future research. Furthermore, the algorithm proposed in this paper is the precondition to identify arrhythmia. And in current practice, only premature beat detection of several data has been studied.
And more researches on the application of this paper will be done in the future.

\section{Acknowledgements}

This work has been partially supported by Zhejiang Key Research and Development Program of Zhejiang Science and Technology Bureau under Grant No.2016CSA160100.

\section{References}

[1] Aiboud Y, El Mhamdi J, Jilbab A, Sbaa H. Review of ecg signal de-noising techniques. Proceedings of 2015 Third IEEE World Conference on Complex Systems (WCCS). 2015.

[2] Joy JM, P. Wavelet based emg artifact removal from ecg signal. Journal of Engineering, Computers \& Applied Sciences. 2013;2(8):4.

[3] Christov I, Neycheva T, Schmid R. Fine tuning of the dynamic low-pass filter for electromyographic noise suppression in electrocardiograms. Computing in Cardiology. 2017;44.

[4] Sayyad RA, Mundada K. Enhancement and denoising of ecg signal using extended kalman filter and extended kalman smoother. Journal of Innovation in Electronics and Communication Engineering. 2016;6(1):22-6.

[5] Oikonomou VP, Tzallas AT, Fotiadis DI. A kalman filter based methodology for eeg spike enhancement. Comput Methods Programs Biomed. 2007;85(2):101-8.

[6] Liu X, Sima J, Huang Y, Liu X, Zhang P. A simplified kalman filter for integrated navigation system with lowdynamic movement. Mathematical Problems in Engineering. 2016;2016:1-9.

[7] Kalman R. A new approach to linear filtering and prediction problems. Journal of Basic Engineering. 1960;82:11.

[8] Goldberger AL, Amaral LA, Glass L, Hausdorff JM, Ivanov PC, Mark RG, et al. Physiobank, physiotoolkit, and physionet: Components of a new research resource for complex physiologic signals. Circulation. 2000;101(23):E215-20.

[9] Moody GB, Muldrow WK, Mark RG, editors. Noise stress test for arrhythmia detectors. Computers in Cardiology $1984 ; 11: 381-4$.

[10] Zidelmal Z, Amirou A, Adnane M, Belouchrani A. Qrs detection based on wavelet coefficients. Comput Meth Prog Bio. 2012;107(3):490-6.

Address for correspondence:

Meng Chen.

Building No.5, East Software Park, No.90 Wensan Road, Hangzhou, Zhejiang, China.

chchenmeng@gmail.com. 